\title{
WEB SERVICES OF MMS APPLICATIONS ON MOBILE DEVICES
}

\section{Cheng-Chih Yang}

Department of Information Engineering, Nan-Kai Institute of Technology, Nan-Tou, Taiwan., czyang@nkc.edu.tw

Cheng-Min Lin

Department of Computer and Communication Engineering, Nan-Kai Institute of Technology, Nan-Tou, Taiwan.

Follow this and additional works at: https://jmstt.ntou.edu.tw/journal

Part of the Computer Engineering Commons

\section{Recommended Citation}

Yang, Cheng-Chih and Lin, Cheng-Min (2006) "WEB SERVICES OF MMS APPLICATIONS ON MOBILE DEVICES," Journal of Marine Science and Technology. Vol. 14: Iss. 3, Article 5.

DOI: $10.51400 / 2709-6998.2070$

Available at: https://jmstt.ntou.edu.tw/journal/vol14/iss3/5

This Research Article is brought to you for free and open access by Journal of Marine Science and Technology. It has been accepted for inclusion in Journal of Marine Science and Technology by an authorized editor of Journal of Marine Science and Technology. 


\section{WEB SERVICES OF MMS APPLICATIONS ON MOBILE DEVICES}

Acknowledgements

The authors would like to thank the National Science Council of R.O.C. for financially supporting this research under Contract NSC-93-2213-E-252-005. 


\title{
WEB SERVICES OF MMS APPLICATIONS ON MOBILE DEVICES
}

\author{
Cheng-Chih Yang* and Cheng-Min Lin**
}

Key words: MMS, PDA, WSDL, GPRS.

\begin{abstract}
This study develops the Web services that are applied in Multimedia Message Service (MMS) applications via the Internet and operated by mobile portable devices, such as Personal Digital Assistant (PDA) and cellular phone. First, a MMS system environment and architecture is modeled. Next, the operations and interface of the MMS system is defined by the Web Services Description Language (WSDL) to represent the web services on MMS application system. Finally, two system prototypes are described in this study that includes distance video cam monitor system and GPRS cellular phone MMS sender.
\end{abstract}

\section{INTRODUCTION}

Multimedia Messaging Service (MMS) [1] represents a revolution of the messaging services in the thirdgeneration $(3 \mathrm{G})$ mobile communications. MMS is also an important technology to be ability to send or receive messages comprising a combination of text, sounds, images, audio and video to MMS capable handsets. Multimedia can be divided into real-time and non-realtime services. Additionally, MMS technology is evolving a development path taken from Short Message Service (SMS) [9] via Enhanced Messaging Service (EMS) [3] to the MMS itself. Among the many MMS applications include content delivery [3], streaming service [4], photo summarization [6], and digital images delivery [7]. Although MMS is intended to provide rich set of content to wireless messaging, MMS cannot to provide an efficient multimedia resource discovery. However, how to make an efficient utilization for multimedia messaging service between mobile devices and web servers is an important issue in next step. Hence, we use the technology of Web services based on the current MMS infrastructure to provide more efficient in

Paper Submitted 09/29/04, Accepted 02/17/06. Author for Correspondence: Cheng-Chih Yang. E-mail: czyang@nkc.edu.tw.

*Department of Information Engineering, Nan-Kai Institute of Technology, Nan-Tou, Taiwan.

**Department of Computer and Communication Engineering, Nan-Kai Institute of Technology, Nan-Tou, Taiwan. service discovery and business transactions.

The attention of web services is increasing with the increasing number of mobile devices recently. A typical Web services architecture comprises three entities, including service providers, service requesters, and service brokers [10]. First of all, service providers create Web services for service requesters. Then, these Web services are registered with service brokers, who handle the requests from service requesters and identify published Web services finally provided by service providers. For example, Ouzzani and Bouguettaya provide a query optimization model based on aggregating the quality of Web service parameters of different Web services [8].

Additionally, Web services comprise three important protocols. First, the Simple Object Access Protocol (SOAP) provides a simple and lightweight protocol for communicating between Web services and client applications. Second, Web Services Description Language (WSDL) is an Extensible Markup Language (XML) based language used to describe Web services. Furthermore, Thomas et al. used Petri Nets to model the Web Services Flow [11]. For testing and evaluating Web services, Tsai et al. devised an XML-based Framework that comprises a test master and a test engine [12]. The researcher permits testers to specify test scenarios and cases derived from WSDL specifications. In addition, a three-tier application has front-end clients (such as, browsers), middle-tier application servers (such as, web servers), and back-end databases [2]. Huang [5] in 2004 designed a Web service platform based on the Open Service Access (OSA). Although the platform provides easily to develop the effort of OSA-based applications, OSA-based networks are tightly coupled and such networks offer low programmability and are non-scalable. Hence, we propose a model based on loose coupled networks to provide flexibility and easy integration with appliance control networks.

In light of the above discussion, this work tries to presents a model based on MMS architecture for video delivery system and to define the WSDL document for the system. In addition, two system prototypes, realtime and non-real-time, are also presented in this work. 
Figure 1 illustrates some related to the integration of MMS application environment with mobile communication, including mobile portable devices, the Internet, Web server, multimedia server and Web cam. The remainder of this paper is organized as follows. Section 2 outlines the proposed system architecture, which has a hybrid web server. Section 3 then introduces this Web representation in WSDL. Moreover, two MMS application examples are given in Section 4. Finally, Section 5 draws conclusions.

\section{SYSTEM ARCHITECTURE}

A mobile MMS application system, including mobile hosts, server providers, a multimedia server, and web cams, is constructed as shown in Figure 2. The portable mobile device, cellular phone receives mobile web pages and send multimedia message to web server or other cellular phone. The PDA receives a live video signal from web cam through web server. In this example, the server is used to perform the functions of an application server set its port at 80 and a multimedia server set its port at 6007; such a server is a hybrid web server.

Our system that is a loose coupled network, represented by graph $G=(V, C)$, can be considered as a set $V$ of $n$ fail-stop nodes $P_{i}$, for $0 \leq i \leq n-1$, interconnected by a communication network consisting of a set $C$ of $m$ bidirectional communication channels. No shared memory or common clock exists among these nodes; the communication between the nodes is by message-passing only. The channels are assumed herein to be FIFO. In addition, the channels are assumed herein to be reliable. We assume that a node has one or more processes.

\section{WEB REPRESENTATION}

Web representation is the main objective in this study. This work analyses those operations and protocol parameters in MMS application system. For applying WSDL, a WSDL flow model is used in the proposed system and being described here.

\section{Operations and protocol parameters}

Three operations being exist between application server and mobile device, namely Vision-Receive, Message-Send, and Message-Receive. A user can request that a web site retrieve the vision image from far end. This operation is called Vision-Receive, and is denoted as $O^{r}$. A SOAP lightweight protocol is used to define our function. Subsequently, a mobile user can send a simple or multimedia message to a personal computer

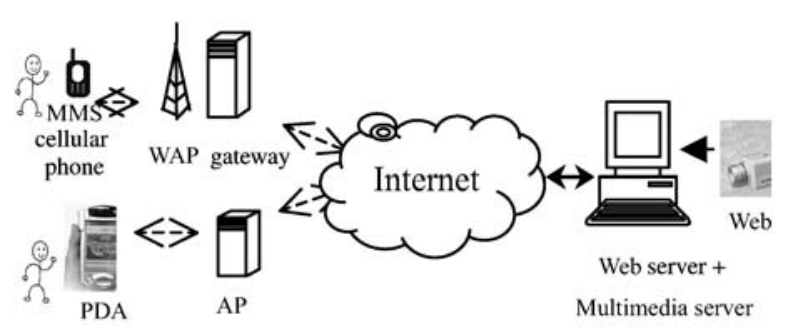

Fig. 1. MMS application environment with mobile communication.

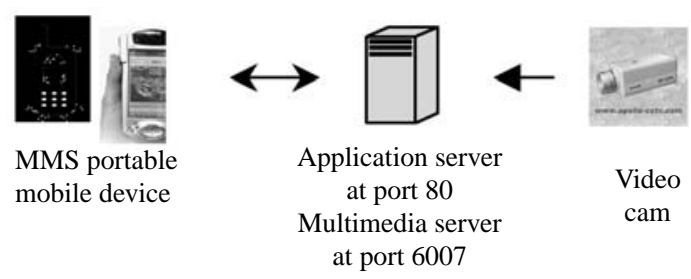

Fig. 2. A hybrid web server architecture.

via a MMS mail server. This control operation is called Message-Send, and is denoted as $O^{s}$. The final operation associated with the data process path is a push operation. Two models can be applied for a mobile system, pull and push. In the push operation, the application server actively sends information to the mobile device of a user. SMS or MMS technology is employed to implement the push mode, is called Message-Receive, and is denoted as $O^{p}$. An operation-based structure is drawn into as block diagrams and shown in Figure 3.

Furthermore, this study defines a mobile control MMS web operation set $O^{m c w}$ which includes three primary functions. $O^{m c w}=\left\{O^{r}, O^{s}, O^{p}\right\}$. For each operation, represents an object embedded with certain parameters, including name, value, and protocol fields. The name field is the object name. This field contains 64 characters and uniquely represents a primary key for this object. The value field is also string type; it can represent message path. Moreover, the protocol field parameter $P^{m}$ is used for application server communication with the client.

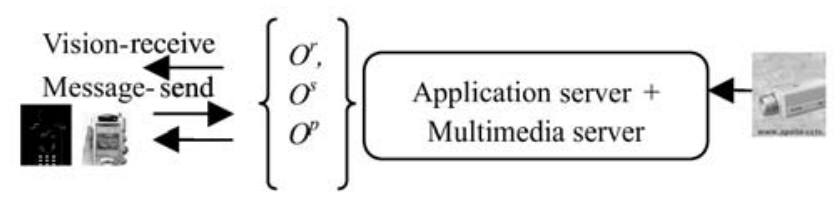

Message-receive

Fig. 3. Operations on MMS application environment. 


\section{WSDL flow model}

WSDL is an XML-based document that includes specific elements: types, message, port-type, binding and service. WSDL is used to describe the capacities of a web service, where it resides, and how to invoke it. This study maps the MMS application to WSDL flow model, and illustrated in Figure 4. This figure contains two ports port-80 and port-6007. The PDA user utilizes MMS protocols to get a distance vision from video cam through a media encoder (function as multimedia server). Another MMS application, a multimedia message is send to the application server from cellular phone in MMS protocols. Actually, a cellular phone user sends a MMS-email to someone. Additionally, a web server also can send a simple message or multimedia message to a cellular phone.

\section{WSDL document}

In previous subsection, the mobile home automation for WSDL flow has been described. In this subsection, an XML-based WSDL is shown in Figure 6. For data type definitions, we define two data types, FrameInquiry and FrameStatus, to be used describe the messages exchanged. FrameInquiry has two parameters, Department and Lab. The former indicates where the location of inquired web camera is and the latter shows the camera is in which lab. Next, both messages are defined for describing the both data being transmitted. Then, a port is shown in Figure 5 of port-c is presented and two messages above are shown here. Furthermore,

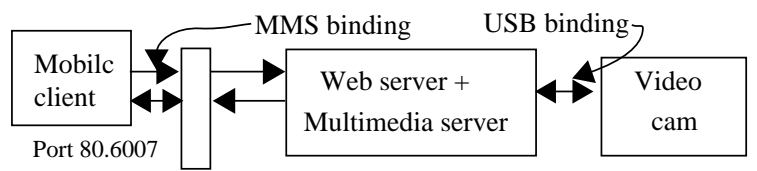

Fig. 4. A MMS application representation in WSDL flow.

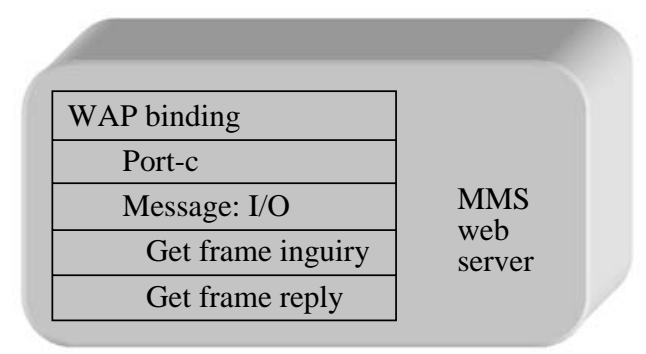

Fig. 5. Implementation of WAPBinding

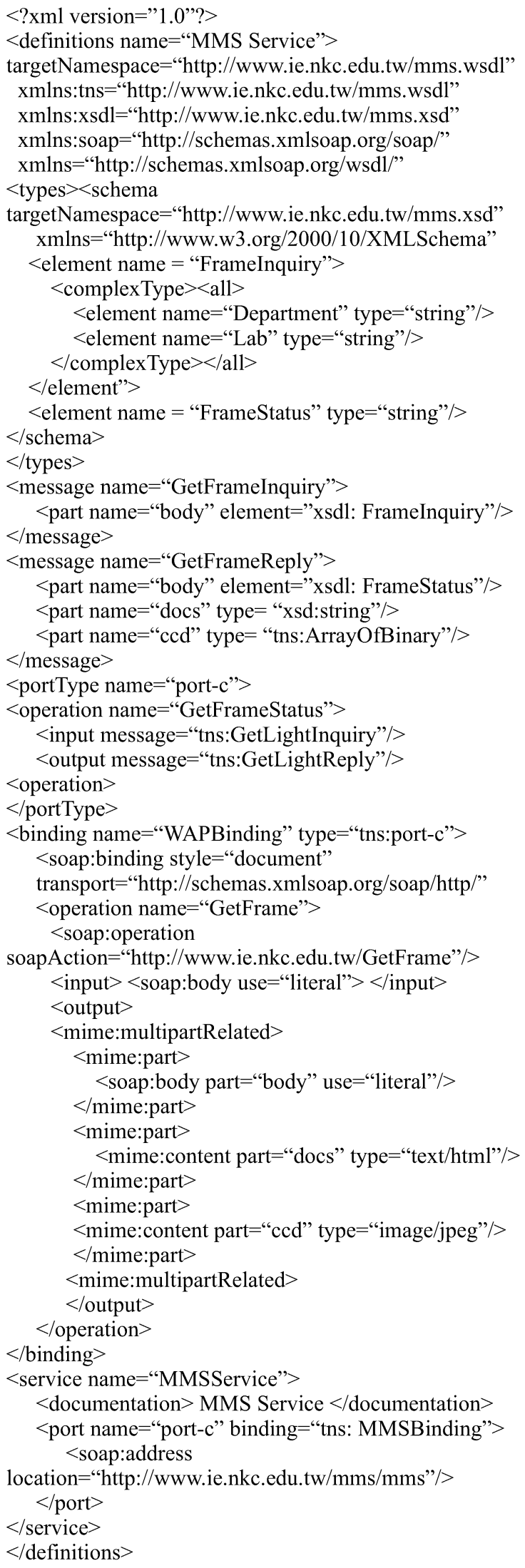

Fig. 6. WSDL document for multimedia service. 
soap binding and specified document style is binding to the port-c. Finally, a web service is defined and the service uses the port-c and port-s. Notice that the definition of about port-s only presents the definition of web services as shown in Figure 6. The tag, <mime: multipartRelated $>$, aggregates an arbitrary set of MIME formatted parts into one message. Notice that a $\langle\mathrm{ccd}\rangle$ tag is used to display a image with jpeg format on a mobile devices.

\section{Advantages of our model}

As above discussion, one advantage of our model is simplification of management of highly customized services because several application servers that are designed by embedded systems act services providers. Furthermore, these application server that are used to control or monitoring appliances are belong to heterogeneous networks. Hence, application servers are hardware dependence but these provide highly customized services.

Another advantage of our model is hardware transparency. We use WDSL documents stored in Web servers to describe providing Web services that are connected by devices. Although these devices are hardware dependency but our Web services are hardware transparency. However, our model is easy integration with foreign existing equipments, such as Web cam.

\section{TWO EXAMPLES}

In this example, an application server is constructed using server operation systems such as Windos 2000 Server or Windos2003 Server, and application software for web pages and databases. A remote appliance monitor is established that includes a control platform MA8-6 that contains multi-type I/O interfaces, DIO, RS232 or RS485, linked to the web appliance server through GPRS protocols. This system can be used to detect earthquakes, stealers, or fires. The appliance server accesses several detector data and saves these data to the public databases to enable application server sharing. The main effect of the application server is to provide html or asp.net webpage for supplying an ideal interface between the web database and the cellular phone user. The user can enquire about and control the appliances from their cellular phone through the WAP or HTTP protocol. Figure 7 illustrates a sensor network that contains anti-fire, anti-earthquake and anti-stealing circuit-broad, and the GPRS control platform, MA8-6. Finally, Figure 8 shows the states of the appliances listed in the database using an IE browser.

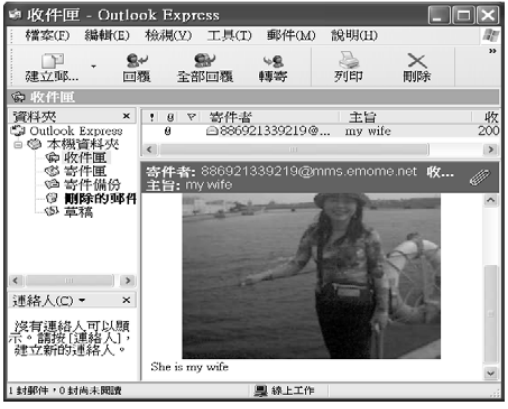

(a) Outlook Express

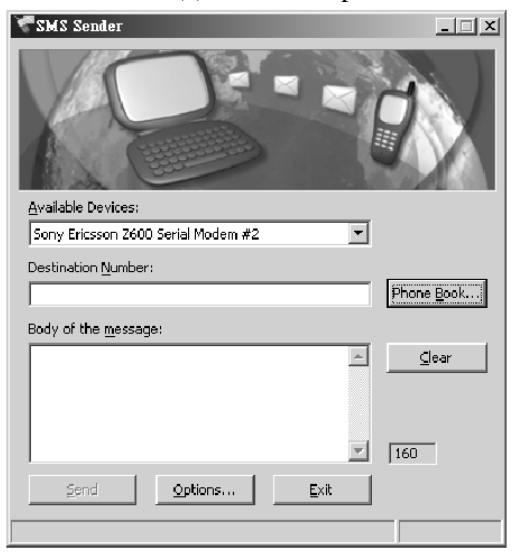

(b) SMS sender.

Fig. 7. An MMS example for multimedia messages.

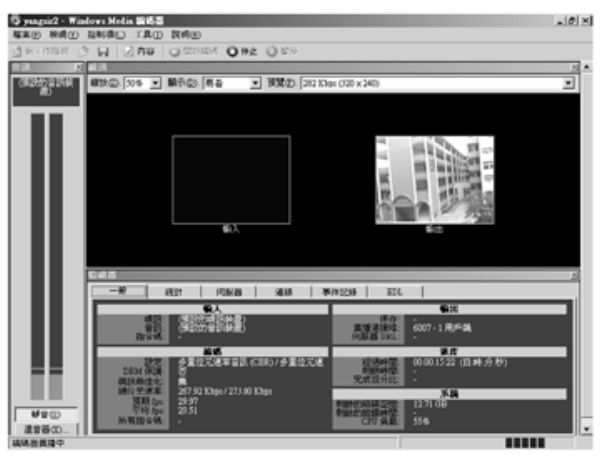

(a) A Window Media Encoder fetches a live view.

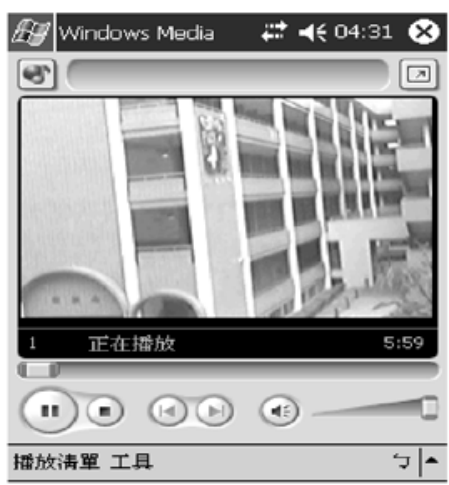

(b) A video response on PDA using MMS protocol.

Fig. 8. An MMS example of video cam monitoring system. 


\section{CONCLUSIONS}

This study addresses MMS application of web services in WSDL representation related with mobile devices. This study also hypothesizes that can be treated as a five-tier architecture for modeling the relation between Web services and mobile home automation. Both servers provide Web services for appliance controls. They fulfill important roles in the proposed system. The application server acts as a service broker, the appliance server acts as a service provider, and a mobile client, PDA or mobile phone acts a service request. Consequently, the architecture of Web services for mobile home automation is made. Additionally, the GPRS mechanism and protocol of WAP can be used to fulfill real-time monitoring functions for remote monitoring. Finally, a small Web service for a sensor network is implemented.

\section{ACKNOWLEDGMENT}

The authors would like to thank the National Science Council of R.O.C. for financially supporting this research under Contract NSC-93-2213-E-252-005.

\section{REFERENCES}

1. Coulombe, S. and Grassel, G., "Multimedia Adaptation for the Multimedia Messaging Service," IEEE Communications Magazine, Vol. 42, No. 7, pp. 120-126 (2004).

2. Frolund, S. and Guerraoui, R., "e-Transactions: End-toEnd Reliability for Three-Tier Architecture," IEEE Transactions on Software Engineering, Vol. 28, No. 4, pp. 378-395 (2002).

3. Gratschew, S., Raitaniemi, J., Ylinen, J., and Loula, P., "A Multimedia Messaging Platform for Content Delivering," Proceedings of the $10^{\text {th }}$ International Conference on Telecommunications, French Polynesia, Vol. 1, pp. 431-435 (2003).

4. Hong, J., Sung, H., Song, J., and Han, S., "Optimization
Scheme for Streaming Service Using Coordination Agent in Multimedia Messaging Service Environment," Proceedings of IEEE Workshop on Software Technologies for Future Embedded Systems, Japan, pp. 43-46 (2003).

5. Huang, H.J., "A Web-Based Service Developing and Executing Platform Using OSA," Master Thesis, Department of Computer Science and Information Engineering, National Cheng Kung University, Taiwan (2004).

6. Li, J., Lim, J.H., and Tian, Q., "Automatic Summarization for Personal Digital Photos," Proceedings of the 2003 Joint Conference of the Fourth International Conference on Information, Communications and Signal Processing, Singapore, Vol. 3, pp. 1536-1540 (2003).

7. Minelli, S.H. and de Polo, A., "Innovative Channels for Digital Images Delivery: Multimedia Messaging Service Technology Enhancing Picture Messaging for Mobile and Internet Environments," IEEE Transactions on Consumer Electronics, Vol. 4, pp. 841-845 (2003).

8. Ouzzani, M. and Bouguttaya, A., "Efficient Access to Web Services," IEEE Internet Computing, Vol. 8, No. 2, pp. 33-34 (2004).

9. Pen, J.Y., Lee, W.T., and Huang, N.F., "Providing Multicast Short Message Services over Self-Routing Mobile Cellular Backbone Network," IEEE Transactions on Vehicular Technology, Vol. 52, No. 1, pp. 240253 (2003).

10. Roy, J. and Ramanujan, A., "Understanding Web Services," IT Professional, Vol. 3, No. 6, pp. 69-73 (2001).

11. Thomas, J.P., Thomas, M., and Ghinea, G., "Modeling of Web Services Flow," Proceedings of IEEE International Conference on E-Commerce, CA, pp. 391-398 (2003).

12. Tsai, W.T., Paul, R., Song, W., and Cao, Z., "Coyote: An XML-Based Framework for Web Service Testing," Proceedings of the $7^{\text {th }}$ IEEE International Symposium on High Assurance Systems Engineering, Japan, pp. 173174 (2002). 